12. Honda, M., Miura, K., Kuratsu, J., and Hayashi, H.: Characterization of a macrophage chemotactic lymphokine produced by purified protein derivative stimulation in vitro and in vivo. Cell Immunol., 67: 213 (1982).

13. Horwitz, D. A. and Garrett, M. A.: Use of leukocyte chemotaxis in vitro to assay mediators generated by immune reactions: I. quantitation of mononuclear and polymorphonuclear leukocyte chemotaxis with polycarbonate (Nuclepore) filters. J. Immunol., 106: 649 (1971).

14. Kahn, C. R.: Insulin resistance, insulin insensitivity, and insulin unresponsiveness: a necessary distinction. Metabolism, 27: 1893 (1978).

15. Matsumoto, T., Gotoh, Y., Narukami, H., Miyamoto, Y., and Honda, M.: Case report: a hyperimmunoglobulin $\mathrm{E}$ syndrome with serum inhibitor against immune functions. Ann. Allergy, 46: 86 (1981).

16. Min-Fu Tsan and Denison, R. C.: Oxidation of a formyl methionyl chemotactic peptide by human neutrophils. J. Immunol., 126: 1387 (1981)

17. Nunoi, $H$, Endo, $F$. Chikazawa, $S$. Namikawa, $T$, and Matsuda, I.: Chemotactic receptor of cord blood granulocytes to the synthesized chemotactic peptide N-formyl-Methonyl-Leucyl-Phenylalanine. Pediatr. Res., 17: 57 (1983).

18. Pincus, S. H., Thomas, I. T., Clark, R. A., and Ochs, H. D.: Defective neutrophi chemotaxis with variant icthyosis, hyperimmunoglobulinemia $\mathrm{E}$ and recurrent infections. J. Pediatr., 87: 908 (1975).

19. Quie, P. G., White, J. G., Holmes, B., and Good, R. A.: In vitro bactericidal capacity of human polymorphonuclear leukocytes: diminished activity in chronic granulomatous disease of childhood. J. Clin. Invest., 46: 668 (1967)

20. Territo, M. C. and Cline, M. J.: Monocyte function in man. J. Immunol, 118 . 187 (1977).

21. Van Scoy, R. E., Hill, H. R., Ritts, R. E. Jr., and Quie, P. G.: Familial neutrophil chemotaxis defect, recurrent bacterial infections, mucocutaneous candidiasis and hyperimmunoglobulinemia E. Ann. Intern. Med., 82: 766 (1975).

22. Ward, P. A., Lepow, I. H., and Newman, L. J.: Bacterial factors chemotactic for polymorphonuclear leukocytes. Am. J. Pathol., 52: 725 (1968).

23. Request for reprints should be addressed to: Dr. Shoji Chikazawa, Department of Pediatrics, Kumamoto University, Medical School, Kumamoto 860 Japan.

24. Received for publication December 29, 1982.

25. Accepted for publication July 5,1983 .

\title{
Cardiac Function and Myocardial Metabolism during Tachycardia Induced by Atrial and Ventricular Pacing
}

\author{
KATSURO IGARASHI ${ }^{(10)}$ \\ Department of Pediatrics, Hirosaki University, School of Medicine, Hirosaki, Japan
}

\section{Summary}

We studied atrial and ventricular pacing-elicited tachycardia in mongrel adult dogs to evaluate ensuing changes in cardiac function and myocardial metabolism. The aortic pressure, left ventricular pressure, and left ventricular end diastolic pressure were measured under anesthesia. Using an electromagnetic flowmeter, coronary blood flow and aortic blood flow were recorded simultaneously with the recording of ECG. Atrial and ventricular pacing (1.5 times the basic heart beat) were performed using a catheter introduced via the jugular vein and positioned in the coronary venous sinus.

Aortic blood and coronary venous blood were sampled for determination of blood oxygen concentrations and lactic acid concentrations. The results showed that oxygen extraction changed little compared with the control study on atrial pacing (AP) and ventricular pacing (VP) whereas the lactate uptake showed little change in the AP control study but decreased slightly in the VP study. It appears that aerobic metabolism occurs during both AP and VP when the pacing rate is not more than 1.5 times the basic heart beat.

\section{Abbreviations}

ABF, aortic blood flow

AP, atrial pacing

CBF, coronary blood flow

HR, heart rate

LVEDV, left ventricular end diastolic volume

PAO, aortic pressure

PLv, left ventricular pressure
Plved, left ventricular end diastolic pressure VP, ventricular pacing

Along with the advances in the treatment and diagnosis of heart diseases, there has been progress in artificial pacemakers. The pacemaker is now used frequently for the treatment and prevention of arrhythmia in both children and adults. Although previous studies have shown clearly that AP is more physiologic and desirable than VP, there are some cases which necessitate the use of VP because of the condition of the illness $(1,4,6)$. There is little opportunity to investigate clinically a tachycardiac state that is maintained by AP or VP for an adequate length of time. An attempt to learn to what extent tachycardia can be maintained by AP or VP, even though nonphysiologic, is by no means useless. From such a viewpoint, we induced experimentally tachycardia by AP and VP in mongrel adult dogs and studied changes in cardiac function and myocardial metabolism.

\section{SUBJECTS AND METHODS}

Ten mongrel dogs weighing 14-24 $\mathrm{kg}$ were anesthetized by intravenous injection of pentobarbital $(30 \mathrm{mg} / \mathrm{kg})$. After intratracheal intubation, a ventilator was used to achieve a constant $\mathrm{pH}$ and $\mathrm{PO}_{2}$ in arterial blood.

With the animals fixed in a supine position, a median incision was made. The pericardial membrane was cut widely from the center to the apex. As shown in Figure 1, a catheter was introduced from the femoral artery and positioned in the arch of the aorta and left ventricle. The catheter was to a Statham $P_{23} D_{b}$ transducer for measuring the $P_{A O}, P_{L V}$, and $P_{L V E D}$. 


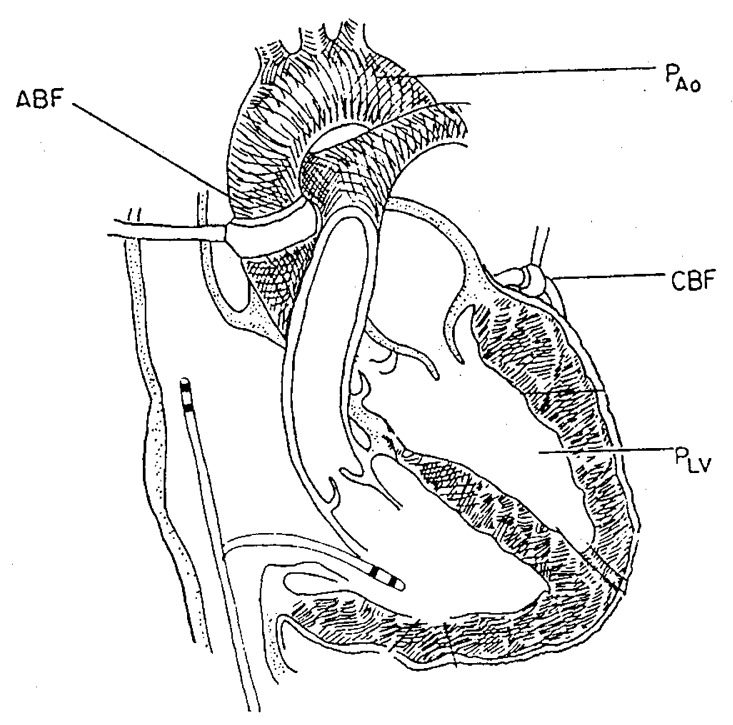

Pacina Cotheter

Fig. 1. A catheter introduced from the femoral artery was positioned in the arch of aorta and left ventricle and connected with the Statham $\mathrm{P}_{23} \mathrm{D}_{\mathrm{b}}$ transducer for measuring the aortic pressure $\left(\mathrm{P}_{\mathrm{AO}}\right)$, left ventricular pressure $\left(P_{L V}\right)$ and left ventricular end diastolic pressure $\left(P_{L V E D}\right)$.

Using an electromagnetic flowmeter placed at the proximal end of both the aorta and the anterior descending branch of the left coronary artery, the $\mathrm{ABF}$ and $\mathrm{CBF}$ were recorded simultaneously with the recording of electrocardiogram. In addition, a catheter introduced from the jugular vein was positioned in the coronary venous sinus, while a pacing catheter was positioned in the right atrium or right ventricle depending on the experimental condition. In the right atrium, the pacing was positioned high on the side wall and in the right ventricle at the apex. As a control study, measurements were taken before pacing. The intensity of pacing was 2 times the threshold for the sensitivity, and the duration was set as $2 \mathrm{~ms}$. The pacing rate was set as 1.5 times the basic heart beat and the duration was $5 \mathrm{~min}$. Immediately before completion of the pacing, aortic blood and coronary venous blood were sampled simultaneously for the determination of blood oxygen concentrations and lactic acid concentrations and, also, for the calculation of oxygen extraction and lactate uptake.

\section{RESULTS}

Cardiac function. Figure 2 shows results obtained from the control study (left) and on AP (right). $P_{\mathrm{LV}}$ showed little change compared with the control. As for $\mathrm{P}_{\mathrm{AO}}$, the systolic pressure showed no change, but the pulse pressure decreased slightly. ABF showed little change. Figure 3 shows the cardiac function on VP. $\mathrm{P}_{\mathrm{AO}}$ and $\mathrm{P}_{\mathrm{LV}}$ decreased about $20 \%$. $\mathrm{P}_{\mathrm{LVED}}$ showed little change. lows:

Oxygen extraction. Oxygen extraction was determined as fol-

Aortic blood oxygen concentration - coronary venous blood

oxygen concentration/aortic blood oxygen concentration

A comparison was made between the oxygen extraction in the control study (before pacing) and during pacing. The results are shown in Figure 4. The oxygen extraction showed little change during AP but rose slightly during VP compared with that before pacing. There was no significant difference $(P<0.05)$ in the rate of elevation between AP and VP.

Lactate uptake. The lactate uptake was determined as:

Aortic blood lactic acid - coronary venous blood lactic acid/ aortic blood lactic acid

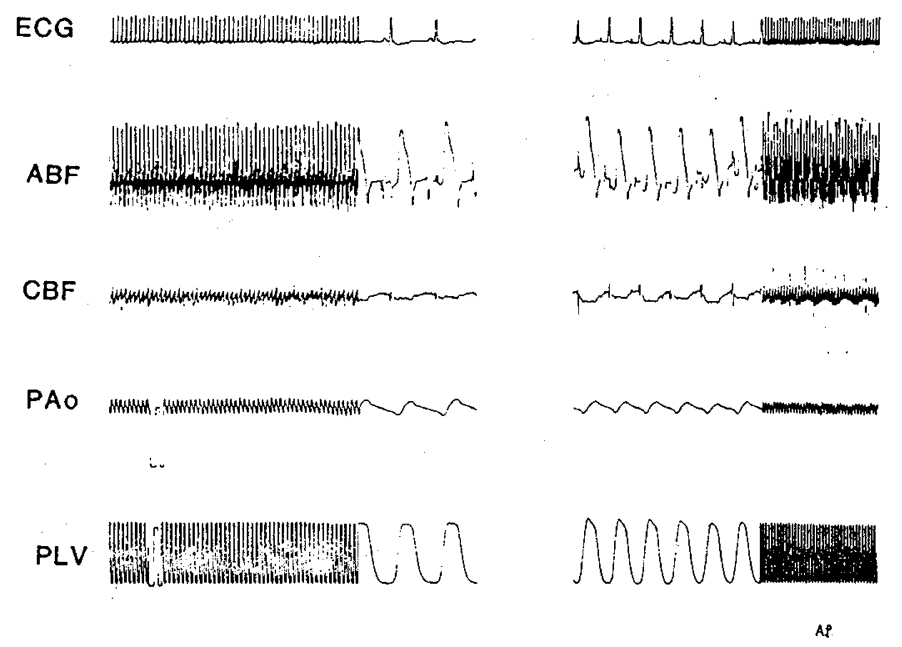

Fig. 2. Results obtained from the control (left) and during AP (right).

ECG

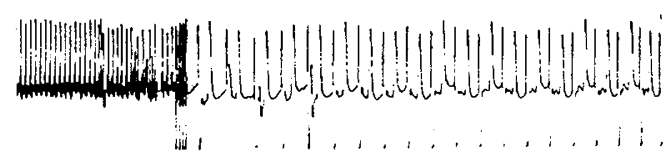

$\mathrm{ABF}$

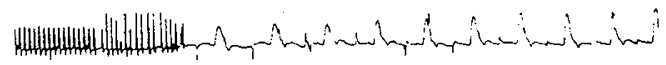

CBF

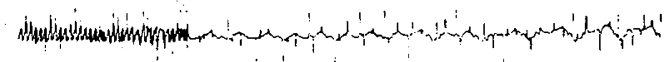

PAO

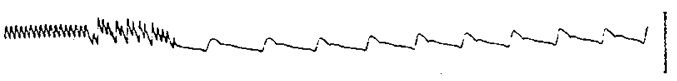

PLV

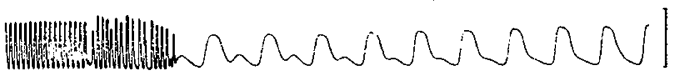

Fig. 3. Cardiac function during VP.

oxygen extraction

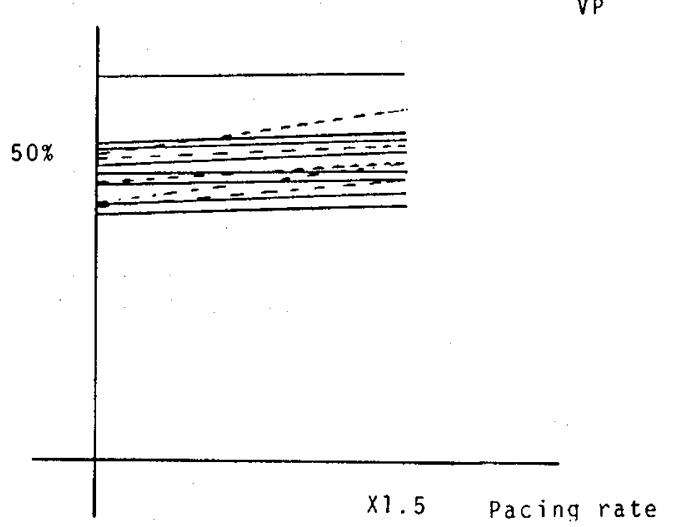

Fig. 4. Oxygen extraction showed little change during AP but rose slightly during VP compared with that before pacing.

Results are shown in Figure 5. During AP, the lactate uptake showed little change or rose slightly compared with the control; however, during VP, it decreased in most cases. 
Lactate uptake

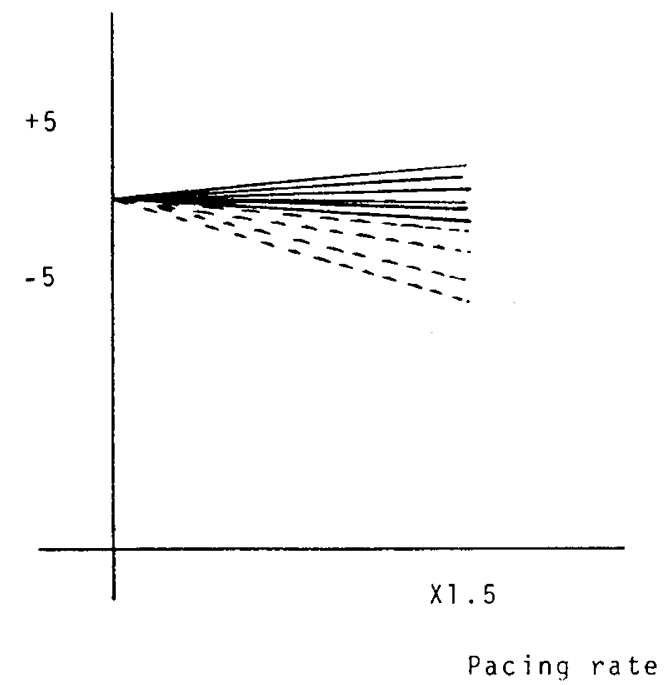

Fig. 5. During AP, the lactate uptake showed little change or rose slightly compared with the control. During VP, it decreased in most cases compared with the control.

\section{DISCUSSION}

The clinical use of either AP or VP depends on the condition of the illness. AP is useful when there is sinus arrest or atrioventricular dissociation whereas VP is effective for the prevention or treatment of ventricular extrasystole and other stimulus abnormalities that occur after operation (9). At the conventional pacing rate no difference in cardiac function is seen between these two methods of pacing. It is interesting to consider what changes in cardiac function and myocardial metabolism will be brought about by tachycardia, and to what extent of tachycardia will the aerobic metabolism be possible. To study these questions, we conducted experiments using mongrel adult dogs.

The basic heart beat of the experimental dogs was 90-120 beat $/ \mathrm{min}$. As the pacing rate by the overdrive showed individual differences, a comparison was made at a fixed rate for the sake of convenience. The pacing rate of 1.5 times the basic heart beat was chosen because the Wenckbach phenomenon occurred when the pacing rate was 2 times the basic heart beat. $P_{A O}, P_{L V}, P_{L V E D}$, $\mathrm{ABF}$, and $\mathrm{CBF}$ were used as indicators of cardiac function.

The double product is the product of the HR and $\mathrm{P}_{\mathrm{Lv}}$; therefore, $H R$ as reflected by the pacing rate is influenced by $P_{L v}$. The cardiac output is the product of the stroke volume and HR (2). In this case, too, stroke volume may be replaced by ABF. From the above equations, it follows that the double product rises at $1.5 \times 1.0=1.5$ compared with the control because $\mathrm{P}_{\mathrm{LV}}$ remains unchanged during $\mathrm{AP}$, and rises slightly at $1.5 \times 0.8=1.2$ because $P_{L V}$ decreases about $20 \%$ during VP. Likewise, cardiac output rises during AP and slightly rises during VP.

Oxygen supply is important for myocardium metabolism. The relationship may be expressed as oxygen supply $=(A-V)$ oxygen dissociation $\times \mathrm{CBF}$. As for the oxygen extraction, the relationship may be expressed as: oxygen extraction $=(\mathrm{A}-$ V) $\mathrm{O}_{2} / \mathrm{AO}_{2}(2,3,8)$. In the present experiment, the oxygen extraction showed little change or rose slightly during both AP and VP. This means that the oxygen supply is influenced mainly by $\mathrm{CBF}$. CBF showed little change during AP but decreased during VP compared with the control. This suggests that the oxygen supply is insufficient during VP. As for the oxygen demand, the relationship is expressed as the oxygen demand = $P_{L V} \cdot$ HR . LVEDV $(2,8)$. LVEDV may be replaced by $P_{L V E D}$ and showed little change on AP and VP; thus $P_{L V}$ and HR may be used as the parameters for the oxygen demand before and after pacing. After pacing, HR was 1.5 times the basal rate whereas $\mathrm{P}_{\mathrm{LV}}$ hardly changed during AP and decreased about $20 \%$ during VP compared with the control. Mathematically, this means that the oxygen demand increased about 1.5 times during AP and about 1.2 times during VP compared with the control.

Usually, the oxygen supply and the oxygen demand are well balanced or at least there is an adequate oxygen supply in the myocardium. When the oxygen supply exceeds the oxygen demand the metabolism becomes aerobic; conversely, the metabolism becomes anaerobic when the oxygen demand exceeds the oxygen supply.

The lactate uptake indirectly reflects the oxidated state of the NAD system. That is, a rise in the lactate uptake indicates a condition of aerobic metabolism. Under aerobic conditions, the lactate uptake does not decrease. A fall in the lactate uptake suggests an anaerobic state $(5,7)$. It appears that aerobic metabolism occurred during AP because the lactate uptake hardly changed or rose slightly.

The oxygen supply presumably does not go below the oxygen demand with the pacing rate about 1.5 times the basic heart beat, although CBF remains almost unchanged. During VP, however, lactate uptake decreased slightly. This suggests that the oxygen demand exceeds the oxygen supply. As evidence to substantiate this speculation, mention is made of a decrease in CBF as the oxygen supply increased during VP. This CBF is proportional to the mean aortic pressure. During VP, the ventricle is not completely filled because of insufficient contraction of the atrium. Furthermore, changes in the mode of intraventricular conduction of excitation exert an influence on the atrial contraction resulting in a decrease in cadiac output. Consequently a decrease in $\mathrm{P}_{\mathrm{LV}}$ and $\mathrm{P}_{\mathrm{AO}}$ cause a decrease in $\mathrm{CBF}$, eventually leading to a decrease in the oxygen supply.

In the present study, the oxygen demand was 1.2 times the control. This probably caused an oxygen debt because the oxygen supply decreased over $20 \%$ compared with the control. Accordingly, whether or not the aerobic metabolism takes place from the aspect of the myocardial metabolism during AP and VP appears to depend on the balance between the degree of reduction in both $P_{L V}$ and CBF. Measuring the blood pressure during AP or VP and using the mean aortic pressure as a guideline are important clinically because measuring CBF at bedside is difficult. At least in this experiment, the pacing rate 1.5 times the basic heart beat led to aerobic metabolism during AP and anaerobic metabolism during VP from the aspect of the myocardial metabolism; therefore, the aerobic metabolism may take place at a pacing rate not more than 1.5 times the basic heart beat. Our experiments were performed under anesthesia and the duration of pacing was set as $5 \mathrm{~min}$. Before AP and VP are applied clinically in the future, it is necessary to conduct studies under various experimental conditions.

\section{REFERENCES AND NOTES}

1. Bechimol A., Ellis, J. G., and Diamond, E. G.: Hemodynamic consequences of atrial and ventricular pacing in patients with normal and abnormal beats: effect of exercise at a fixed atrial and ventricular rate. Am. J. Med., 39: 911 (1965).

2. Bern, R. and Levy, M.,: Cardiovascular Physiology. 3rd ed. p. 107 (Mosby, St. Louis, 1977).

3. Braunwald, E., Ross, R., and Sonnenblick E.: Mechanism of Contraction of the Normal and Failing Heart. p. 204 (Little, Brown Co., Boston, MA, 1976).

4. Gilmore, J. P.: Synchronicity of ventricular contraction: observation comparing hemodynamic effects of atrial and ventricular pacing. Br Heart J., 25: 299 (1963).

5. Ishikawa K.: A Textbook of Advanced Clinical Cardiology. (Igaku Shoin, Tokyo, 1979).

6. Kawamura, T., Koyanagi, H., and Hashimoto A.; A hemodynamic comparison of ventricular and atrial pacing after open-heart surgery. Heart, 10:140 (1978).

7. Murao, S. and Sugishita, Y.: Cardiac function under myocardial ischemia. Jpn. Heart J., 18: 366 (1977).

8. Rushmer, R. F.: Cardiovascular Dynamics. (W. B. Saunders Co., Philadelphia, PA 1976). 
9. Woodson, R. D. and Starr, A.: Atrial pacing after mitral valve surgery. Arch. Surg., 97: 984 (1978).

10. Requests for reprints should be addressed to: Dr. Katsuro Igarashi, Department of Pediatrics, Hirosaki University, School of Medicine, Hirosaki University,
Hirosaki, Japan.

11. Received for publication November $25,1982$.

12. Accepted for publication June 23, 1983.

\title{
Increase of Tryptophan and 5-Hydroxyindole Acetic Acid in the Brain of Ornithine Carbamoyltransferase Deficient Sparse-Fur Mice
}

\author{
C. BACHMANN ${ }^{(25)}$ AND J. P. COLOMBO \\ Department of Clinical Chemistry, Inselspital, University of Bern, Bern, Switzerland
}

\begin{abstract}
Summary
Sparse-fur mice, $28 \mathrm{~d}$ of age with the $\mathrm{x}$-chromosomal inherited defect of ornithine carbamoyltransferase, were used to investigate if tryptophan and the serotonin pathway in the brain are affected in this animal model which closely resembles the human inborn error of metabolism. Increased concentrations of tryptophan and 5-hydroxyindole acetic acid were found in forebrain and brainstem. Application of probenecid, which blocks the efflux of 5 hydroxyindole acetic acid from the brain, led to an augmented accumulation of this serotonin metabolite in the affected males. We conclude that the increased concentration of tryptophan in brain and the subsequent increased flux through the serotonin pathway are a consequence of hyperammonemia in this inherited defect of urea synthesis. Surprisingly, increased carbamoylphosphate synthetase was found in the liver of the male sparse-fur mice.
\end{abstract}

\section{Abbreviations}

CPS, carbamoylphosphate synthetase

HIAA, 5-hydroxyindole acetic acid

HT, 5-hydroxytryptamine

NAGS, $\boldsymbol{N}$-acetylglutamate synthetase

OCT, ornithine carbamoyltransferase

spf, sparse-fur

TRP, tryptophan

Sparse-fur (spf) mice have a $\mathrm{x}$-chromosomal inherited defect of OCT (E C 2.3.3.11). In affected males this leads to hyperammonemia $(10,17)$ as in the human variant of OCT deficiency, an inborn error of metabolism which presents with neurologic symptoms $(1,23)$. These are presumably mediated by changes of neurotransmitters in brain. We chose to investigate metabolites of the serotonin pathway in spf mice, because TRP and HIAA were increased in two animal models where hyperammonemia is present. Studies with rats subjected to portacaval shunting have repeatedly led to the conclusion that chronic liver insufficiency (with hyperammonemia) increases the brain concentration of TRP, the precursor of serotonin (HT), and of HIAA, the main metabolite of HT (11). Because of the multiple metabolic changes encountered in portacaval anastomosis, the particular role of hyperammonemia has not been clearly delin- eated in that complex model (11). For this reason we used a simpler set-up and injected rats with urease. We found that the transport of TRP into the brain was enhanced and that the concentrations of TRP and HIAA in forebrain and brainstem were higher than in pair-fed controls (3).

The following investigations were performed to find out if an inherited defect of an urea cycle enzyme leads to comparable changes in the serotonin pathway. We examined if, in the spf mouse model, the affected males (spf/Y) had increased concentrations of TRP, HT, and HIAA in brain as compared with their unaffected littermates $(+/ Y)$. In order to detect if the flux through the serotonin pathway is altered in spf mice, the indole metabolites were determined also after injection of probenecid, an inhibitor of HIAA transport out of the brain.

\section{MATERIALS AND METHODS}

Spf-females (spf/+) from the Oak Ridge National Laboratory (Oak Ridge, TN) were mated with C57BL*BALBc males (Jackson Lab, Bar Harbour, ME). The offspings were used for the experiments. These were carried out on affected males (spf/Y) which are readily recognized by their sparse fur and on unaffected males (controls, $+/ \mathrm{Y}$ ) at the age of $28 \mathrm{~d}$. Data on females (both $\mathrm{spf} /+$ and $+/+)$ are also presented. The animals had not yet been weaned. At 8 a.m. the animals were withdrawn from their mother and placed with a nonlactating female mouse in order to obtain metabolite concentrations unbiased by postprandial amino acid alterations. At 10 a.m. physiologic saline or probenecid $(200 \mathrm{mg} / \mathrm{kg}$ body weight) was injected intraperitoneally. The animals were decapitated at 11 a.m. and the brain was dissected on an ice cold glassplate within $3 \mathrm{~min}$ into forebrain and brainstem by a precollicular cut after removing the cerebellum. The brain parts were immediately weighed and homogenized in perchloric acid $(0.1 \mathrm{~mol} / 1 ; 2.0 \mathrm{ml} / 250 \mathrm{mg}$ of brain) containing ascorbic acid $(0.23 \mathrm{mmol} / \mathrm{l})$, EDTA $(3.4 \mathrm{mmol} / \mathrm{l})$, and vanillic acid as internal standard. The deproteinized samples were applied to an Amberlite CG50, type 1, column $(3 \times 10 \mathrm{~mm})$ and the indoles eluted with $3.2 \mathrm{ml} \mathrm{HCl}(1 \mathrm{~mol} / \mathrm{l})$ containing $1 \%$ cystein. The eluate was applied to a SepPakC-18 capsule (Waters Ass., Milford, MA). After washing with $2 \mathrm{ml}$ of bidistilled water the indoles were finally obtained by application of $5.0 \mathrm{ml}$ of sodium acetate/citrate buffer $(0.1 \mathrm{mmol} / 1, \mathrm{pH} 4.3)$ containing $85 \%(\mathrm{v} / \mathrm{v})$ methanol. Ten microliters of the dried extract dis- 